\title{
Lurasidone in Therapy of Treatment-resistant Ultra-rapid Cycling Bipolar Disorder: Case Report
}

\author{
Marcin Siwek ${ }^{1}$, Aleksandra Gorostowicz ${ }^{2}$ \\ ${ }^{1}$ Department of Affective Disorders, Department of Psychiatry, ${ }^{2}$ Department of Psychiatry, Jagiellonian University Medical College, Cracow, Poland
}

\begin{abstract}
Lurasidone is an atypical antipsychotic approved for the treatment of schizophrenia and bipolar depression. It seems to have a favorable metabolic profile and low risk of causing adverse interactions. Here we present a case of a 25-year old female patient with treatment-resistant ultra-rapid cycling bipolar disorder, obesity, hypothyroidism, and epilepsy. Because of predominant depressive symptoms, occasional occurrence of brief psychotic symptoms and patient's somatic comorbidities, treatment with lurasidone was initiated. Clinical improvement was observed 3 weeks and cessation of ultra-rapid cycling course of the disease 8 weeks after the beginning of lurasidone treatment. The patient's level of functioning improved and body mass significantly decreased, with good tolerance of the pharmacotherapy. Lurasidone seems to be a promising treatment option in patients with treatment-resistant rapid cycling bipolar disorder.
\end{abstract}

KEY WORDS: Bipolar disorder; Lurasidone hydrochloride; Obesity; Treatment-resistance.

\section{INTRODUCTION}

Lurasidone is an atypical antipsychotic approved by the Food and Drug Administration for the treatment of schizophrenia and bipolar I depression [1]. Its mechanism of action is based on antagonism of $\mathrm{D}_{2}, 5-\mathrm{HT}_{2 \mathrm{~A}}, 5-\mathrm{HT}_{7}$, and $\alpha 2 \mathrm{~A}$-adrenergic receptors and partial agonism of $5-\mathrm{HT}_{1 \mathrm{~A}}$. It has low affinity for the muscarinic $M 1$, histamine $\mathrm{H} 1$, 5 -HT2C, and $\alpha 1 \mathrm{~A}$-adrenergic receptors [2]. Data from randomized placebo-controlled studies in schizophrenia and bipolar depression indicate that lurasidone was not associated with clinically significant metabolic changes (when compared to placebo) in weight, lipid profile or glycemic control [3-5].

Here we present the case of a female patient with treatment-resistant ultra-rapid cycling (ultra-RC) bipolar disorder (BD) and somatic comorbidities successfully treated with lurasidone. Informed consent was provided by the patient.

Received: October 25, 2020 / Revised: December 15, 2020

Accepted: December 17, 2020

Address for correspondence: Aleksandra Gorostowicz Department of Psychiatry, Jagiellonian University Medical College, Kopernika st. 21 A, Cracow 31-501, Poland

E-mail: ola.gorostowicz@interia.pl

ORCID: https://orcid.org/0000-0002-9945-2378

\section{CASE}

A 25-year-old white Caucasian female patient was admitted to the outpatient psychiatric clinic in September 2018 in order to optimize pharmacotherapy. The patient reported frequent mood changes occurring since she was 8-year-old - she had been experiencing periods of lowered mood, lack of energy, social withdrawal as well as periods of elevated mood and energy level, racing thoughts. The phases sometimes lasted only hours but were intense, with distinct clinical symptoms of depression or hypomania. Usually mood switches were occurring during one day, but periods of altered mood lasting for days or several weeks were also present. The patient had never had any depressive or hypomanic phases lasting longer than 4-5 weeks.

She started psychiatric treatment and individual psychotherapy in 2016 after suicide attempt by overdose of painkillers.

In autumn 2017 the patient was admitted to the outpatient hospital unit for the treatment of mood disorders to verify the diagnosis and modify pharmacotherapy. During the hospitalization she was presenting frequent mental state changes from depressive to hypomanic phases which lasted up to a few days. Euthymic days were sporadic. Magnetic resonance imaging of the head was normal.

(ㄷ) This is an Open-Access article distributed under the terms of the Creative Commons Attribution Non-Commercial License (http://creativecommons.org/licenses/by-nc/4.0) which permits unrestricted non-commercial use, distribution, and reproduction in any medium, provided the original work is properly cited. 
Treatment of hypothyroidism was optimized after endocrinological consultation (at admission thyroid stimulating hormone level was around $17 \mathrm{ulU} / \mathrm{ml}$ ). Neuropsychological assessment did not demonstrate any marked cognitive dysfunctions. The hospitalization did not cause long-term and significant stabilization of the patient's mental state but confirmed the diagnosis of ultra-RC BD.

The patient's medical record included somatic comorbidities: obesity (body mass index [BMI] on admission to the outpatient clinic: $44.6 \mathrm{~kg} / \mathrm{m}^{2}$ ), hypothyroidism, epilepsy with absence seizures (diagnosed in 2017), history of neonatal sepsis, pancreatitis and hepatitis type B (treated with interferon) in early childhood. The patient denied use of alcohol or illegal psychoactive substances.

After admission she was initially treated with valproate $(1,200 \mathrm{mg}$ daily, blood levels were in the therapeutic range), lamotrigine (100 mg daily) and omega-3 fatty acids. The treatment with lamotrigine and valproate was recommended by neurologist as it provided good seizure control and included antiepileptic medications which do not tend to cause deterioration of mental state. Basic laboratory tests were normal. Although adherence to treatment and tolerance of pharmacotherapy were good, the patient complained of frequent mood changes (up to a few switches per week), generalized and social anxiety, anhedonia, insomnia (duration of nighttime sleep was around 4 hours). Treatment with sleep-promoting antidepressant medications (agomelatine $25 \mathrm{mg}$ per day or trazodone controlled release daily dose up to $75 \mathrm{mg}$ ) was not associated with any symptomatic improvement. The patient experienced even a two-week period of worsening of mental state-she had suicidal thoughts and brief auditory hallucinations. Because of the treatment-resistance (previous therapy with different agents [quetiapine, aripiprazole, vortioxetine, lamotrigine, valproate, selective serotonin reuptake inhibitor, olanzapine, risperidone, serotonin norepinephrine reuptake inhibitor, agomelatine, trazodone controlled release] was either unsuccessful or only temporarily effective, with rapid loss of therapeutic action), somatic comorbidities and illness course with predominant depressive symptoms, it was decided to begin treatment with lurasidone (with continuation of pharmacotherapy with valproate and lamotrigine- this antiepileptic treatment was recommended by neurologist due to optimal seizure control acquired with the therapy). The daily dose was gradually increased, starting from $18.5 \mathrm{mg}$ per day. After approximately 3 weeks response to treatment was observed - the patient noticed that mood changes were less frequent and less prominent than before. After 8 weeks (37 mg of lurasidone daily) definite symptomatic improvement was present: the patient reported having whole days of euthymia, auditory hallucinations were occurring rarely.

In the forthcoming months, due to persistent symptoms: inner tension, anxiety, low stress tolerance, auto-aggressive impulses, periodic ideas of reference and sleep problems, treatment was gradually modified - the attending psychiatrist added trazodone in extended release formula up to $300 \mathrm{mg}$ per day, increased the dose of lurasidone to $111 \mathrm{mg}(0-0-111 \mathrm{mg})$. Opipramol and diphenhydramine (100 mg and $50 \mathrm{mg}$ respectively, taken at night only occasionally) were added as sleep promoting agents (previous treatment with zopiclone was unsuccessful). Tolerance of the pharmacotherapy was good. The patient's body mass gradually decreased - her final $\mathrm{BMI}$, at the end of the observation period, was $38.7 \mathrm{~kg} / \mathrm{m}^{2}$ (nearly $20 \mathrm{~kg}$ of weight reduction). During the next months after pharmacotherapy modifications the patient reported significant improvement-her mood was much more stable and reactive, depressive or hypomanic phases were absent, she had no perceptual disturbances or delusional thoughts, she denied suicidal ideations or plans, her general level of functioning was improved. Changes in the results of symptom scales measuring depressive, hypomanic and psychotic symptoms were also observable when comparing patient's assessments before and after a few months of treatment with lurasidone: Quick Inventory of Depressive Symptomatology-Self Report change from 16 to 5 points, Young Mania Rating Scale from 8 to 2 points, and Brief Psychiatric Rating Scale from 35 to 20 points.

After 20 months of observation since the initiation of lurasidone treatment, mood stabilization, clinical and functional improvement and cessation of ultra-RC course of the disease were maintained.

\section{DISCUSSION}

The term "rapid cycling" is one of the specifiers used for BD in the Diagnostic and Statistical Manual of Mental Disorders, 5th edition. It is defined as a course of the illness with at least four affective episodes during a 12-month 
period. Episodes are demarcated by a remission period of at least 2 months (for episodes of the same polarity) or by a switch to an episode of opposite polarity [6]. Some patients experience very short cycles lasting for days (ultra-RC). RC pattern seems to be a transitory phenomenon, for which the lifetime prevalence ranges from 26 to $43 \%$ [7]. The RC course of $\mathrm{BD}$ has been associated with earlier age at onset, higher level of disability, female sex, bipolar type II, more severe depressive symptoms, more anxiety, increased suicide risk, atypical depressive symptoms, higher treatment resistance, more frequent use of psychoactive substances (compared to non-RC bipolar patients) [8-11]. It is hypothesized that RC can be triggered by use of antidepressants or hypothyroidism (including subclinical) [7]. The literature on the pharmacological treatment of RC (both acute and maintenance) is still sparse and of generally poor quality - most of the published data comes from post hoc analysis [12,13]. According to Canadian Network for Mood and Anxiety Treatments guidelines, there is no clear evidence for the superiority of any specific agent to treat manic or depressive symptoms in RC patients-pharmacotherapy should be chosen based on available general recommendations for acute and maintenance treatment in BD [14]. However, data indicate that aripiprazole and olanzapine can be efficacious in the treatment of acute manic symptoms, while quetiapine reduce intensity of depressive symptoms in RC patients. Aripiprazole and olanzapine also seem to be effective in the relapse prevention $[12,13]$. Lithium, divalproex, olanzapine, and quetiapine have been observed to have antidepressive maintenance efficacy. It is possible that for RC patients polytherapy with mood stabilizers, rather than monotherapy, might be necessary [14]. There is currently scarcity of data regarding pharmacotherapy in treatment-resistant RC BD [15].

Patients with RC were permitted in some of the clinical trials on lurasidone treatment in BD [16-18]. However, data on lurasidone efficacy exclusively in the subgroup of RC patients are missing. To the best of our knowledge, there are no published reports of successful treatment with lurasidone as add-on therapy in patients with ultra-RC.

Lurasidone has a favorable metabolic profile $[19,20]-$ the medication did not differ from placebo in terms of weight gain in recent meta-analyses on efficacy and tolerability of antipsychotics in schizophrenia [5,21]. Lurasidone also seems to cause no clinically significant weight or metabolic changes (compared to placebo) in the population of bipolar patients [3]. Moreover, weight loss was observed in patients switched from prior second-generation antipsychotics (with known propensity to cause weigh gain) to lurasidone treatment [22]. Data from clinical trials do not indicate that lurasidone separates from placebo in terms of seizure incidence [23].

Taking into consideration the above mentioned data, in case of our patient lurasidone was the optimal choice due to a few clinical features. Firstly, depressive symptoms were predominant in the course of disease. Secondly, patient's somatic comorbidities (obesity, epilepsy) greatly reduced a number of available, safe treatment options. In the aspect of polytherapy, lurasidone was preferred as it does not affect cytochromes activity and its receptor profile did not cause adverse pharmacodynamic interactions. We assume that lurasidone can be effective in the therapy of treatment-resistant RC BD due to its multimodal profile of receptor activity which can contribute to the wide spectrum of clinical properties: antipsychotic, antidepressive, mood stabilising and potential (but still not well-proven) antimanic.

Although the patient had received different medications with proven efficacy in the treatment of RC BD (for an adequate period of time and in adequate doses), none of them were effective in the longer term. Moreover, the patient was occasionally experiencing brief psychotic or pre-psychotic symptoms like auditory hallucinations or ideas of reference, which made it rational to introduce a medication with both mood stabilizing and antipsychotic action. It is worth noticing that we observed clinical improvement as soon as 3 weeks and cessation of ultra-RC course 8 weeks after the initiation of lurasidone treatment.

Apart from symptomatic and functional improvement, the patient's weight significantly decreased and the treatment tolerance was good (no epileptic seizures were observed during lurasidone treatment).

In conclusion, lurasidone can be a promising treatment option in patients with treatment-resistant RC BD, also with concomitant metabolic diseases and epilepsy.

\section{- Conflicts of Interest}

No potential conflict of interest relevant to this article was reported.

\section{Author Contributions}

Conceptualization: Marcin Siwek, Aleksandra Goros- 
towicz. Data acquisition: Marcin Siwek. Formal analysis: Marcin Siwek, Aleksandra Gorostowicz. Supervision: Marcin Siwek. Writing - original draft: Aleksandra Gorostowicz, Marcin Siwek. Writing - review \& editing: Marcin Siwek, Aleksandra Gorostowicz.

\section{ORCID}

Aleksandra Gorostowicz

https://orcid.org/0000-0002-9945-2378

Marcin Siwek

https://orcid.org/0000-0002-3109-1895

\section{REFERENCES}

1. Loebel A, Citrome L. Lurasidone: a nove/ antipsychotic agent for the treatment of schizophrenia and bipolar depression. BJPsych Bull 2015;39:237-241.

2. Ishibashi T, Horisawa T, Tokuda K, Ishiyama T, Ogasa M, Tagashira R, et al. Pharmacological profile of lurasidone, a novel antipsychotic agent with potent 5-hydroxytryptamine 7 (5-HT7) and 5-HT1A receptor activity. J Pharmacol Exp Ther 2010;334:171-181.

3. Citrome L, Ketter TA, Cucchiaro J, Loebel A. Clinical assessment of lurasidone benefit and risk in the treatment of bipolar I depression using number needed to treat, number needed to harm, and likelihood to be helped or harmed. I Affect Disord 2014; 155:20-27.

4. Loebel A, Cucchiaro J, Silva R, Kroger H, Hsu J, Sarma K, et al. Lurasidone monotherapy in the treatment of bipolar I depression: a randomized, double-blind, placebo-controlled study. Am J Psychiatry 2014;171:160-168.

5. Leucht S, Cipriani A, Spineli L, Mavridis D, Orey D, Richter F, et al. Comparative efficacy and tolerability of 15 antipsychotic drugs in schizophrenia: a multiple-treatments meta-analysis. Lancet 2013;382:951-962.

6. American Psychiatric Association. Diagnostic and statistical manual of mental disorders. 5th ed. Washington, DC:American Psychiatric Association;2013.

7. Carvalho AF, Dimellis D, Gonda X, Vieta E, Mclntyre RS, Fountoulakis KN. Rapid cycling in bipolar disorder: a systematic review. J Clin Psychiatry 2014;75:e578-e586.

8. Gigante AD, Barenboim IY, Dias RD, Toniolo RA, Mendonça T, Miranda-Scippa Â, et al. Psychiatric and clinical correlates of rapid cycling bipolar disorder: a cross-sectional study. Braz J Psychiatry 2016;38:270-274.

9. Hajek T, Hahn M, Slaney C, Garnham J, Green J, Růzicková $\mathrm{M}$, et al. Rapid cycling bipolar disorders in primary and tertiary care treated patients. Bipolar Disord 2008;10:495-502.

10. Lee S, Tsang A, Kessler RC, Jin R, Sampson N, Andrade L, et al. Rapid-cycling bipolar disorder: cross-national community study. Br J Psychiatry 2010;196:217-225.

11. Valentí M, Pacchiarotti I, Undurraga J, Bonnín CM, Popovic
D, Goikolea JM, et al. Risk factors for rapid cycling in bipolar disorder. Bipolar Disord 2015;17:549-559.

12. Fountoulakis KN, Dimellis D. The treatment of rapid cycling bipolar disorder. In: Carvalho AF, Vieta E, editors. The treatment of bipolar disorder: integrative clinical strategies and future directions. Oxford:Oxford University Press;2017. p.65-80.

13. Fountoulakis KN, Kontis D, Gonda X, Yatham LN. A systematic review of the evidence on the treatment of rapid cycling bipolar disorder. Bipolar Disord 2013;15:115-137.

14. Yatham LN, Kennedy SH, Parikh SV, Schaffer A, Bond DJ, Frey $\mathrm{BN}$, et al. Canadian Network for Mood and Anxiety Treatments (CANMAT) and International Society for Bipolar Disorders (ISBD) 2018 guidelines for the management of patients with bipolar disorder. Bipolar Disord 2018;20:97-170.

15. Bazire S. Psychotropic drug directory 2018: the professionals' pocket handbook and aide memoire. London:Lloyd-Reinhold Publications;2018. p.59-60.

16. Ketter TA, Sarma K, Silva R, Kroger H, Cucchiaro J, Loebel A. Lurasidone in the long-term treatment of patients with bipolar disorder: a 24-week open-label extension study. Depress Anxiety 2016;33:424-434.

17. Mclntyre RS, Cucchiaro J, Pikalov A, Kroger H, Loebel A. Lurasidone in the treatment of bipolar depression with mixed (subsyndromal hypomanic) features: post hoc analysis of a randomized placebo-controlled trial. J Clin Psychiatry 2015; 76:398-405.

18. Calabrese JR, Pikalov A, Streicher C, Cucchiaro J, Mao Y, Loebel A. Lurasidone in combination with lithium or valproate for the maintenance treatment of bipolar / disorder. Eur Neuropsychopharmacol 2017;27:865-876.

19. Wichniak A, Dudek D, Heitzman J, Kapłon-Cieślicka A, Mamcarz A, Samochowiec J, et al. Metabolic risk reduction in patients with schizophrenia treated with antipsychotics: recommendations of the Polish Psychiatric Association. Psychiatr Pol 2019;53:1191-1218.

20. Reynolds GP, Dalton CF, Watrimez W, Jackson J, Harte MK. Adjunctive lurasidone suppresses food intake and weight gain associated with olanzapine administration in rats. Clin Psychopharmacol Neurosci 2019;17:314-317.

21. Huhn M, Nikolakopoulou A, Schneider-Thoma J, Krause M, Samara M, Peter N, et al. Comparative efficacy and tolerability of 32 oral antipsychotics for the acute treatment of adults with multi-episode schizophrenia: a systematic review and network meta-analysis. Lancet 2019;394:939-951.

22. Meyer JM, Ng-Mak DS, Chuang CC, Rajagopalan K, Loebel A. Weight changes before and after lurasidone treatment: a realworld analysis using electronic health records. Ann Gen Psychiatry 2017;16:36.

23. Bazire S. Psychotropic drug directory 2018: the professionals' pocket handbook and aide memoire. London:Lloyd-Reinhold Publications;2018. p.255-256. 